\title{
Combining ADF-EDX scattering cross-sections for elemental quantification of nanostructures
}

Zezhong Zhang ${ }^{1}$, Annick De Backer ${ }^{1}$, Ivan Lobato ${ }^{1}$, Sandra Van Aert ${ }^{1}$ and Peter Nellist ${ }^{2}$

${ }^{1}$ EMAT, University of Antwerp, Antwerp, Belgium, Belgium, ${ }^{2}$ University of Oxford, Department of Materials, United States

To make the structure-property relationship in nanostructured materials, we need to probe their crystal structures and compositions at the atomic scale. From a single ADF-STEM image, it has been demonstrated that one can determine the atomic column positions and count the number of atoms for homogenous materials with high precision and accuracy [1-2]. Advanced materials usually consist of multiple elements in a complicated structure and significant difficulties remain to disentangle the contributions of composition and thickness in STEM. To address this challenge, we propose to combine ADF-EDX for the quantification of multiple elements in heterogeneous systems.

Due to the dynamic nature of electron scattering, ADF and EDX have a non-linear relationship against thickness and composition, particularly at the atomic scale in a zone orientation. However, preliminary experiments indicate a linear dependence between EDX and ADF scattering cross-sections, which is defined as the integration of signal intensities for each atomic column. As shown in Fig. 1, we performed multislice frozen phonon calculations for ADF and EDX simultaneously for a pure Au crystal and confirm such linearity of EDX against ADF with a high inner collection angle, which is a result of the incoherent nature of both signal modes.

Because of the incoherent signal mode, we can treat dynamical scattering as a superposition of individual atoms focussing the incident electrons. Here we expanded the so-called atomic lensing model [3] (developed previously for ADF) to spectroscopy. For a mixed column, the ordering possibilities grow exponentially with the thickness and types of elements thus quickly exceeding the capability of multislice calculations due to computational cost. In contrast, the atomic lensing model allows fast generation of EDX scattering cross-sections with the ordering of elements taken into account under the channelling condition. As shown in Fig. 2 for a core-shell Au-Pt nanorod, scattering cross-sections extracted from the multislice calculations agree reasonably well with the atomic lensing model predictions but are very different from those of the linear model where the signal is assumed to scale linearly with the number of atoms for each type. To deploy the atomic lensing model to experimental results, we can incorporate the experimentally measured EDX partial cross-section [4], which is called partial since it includes all the microscope-dependent factors during normalisation thus bypassing the difficult characterisation of the EDX detector. This method allows us to explore the enormous ordering possibilities of heterogeneous materials with multiple elements. 


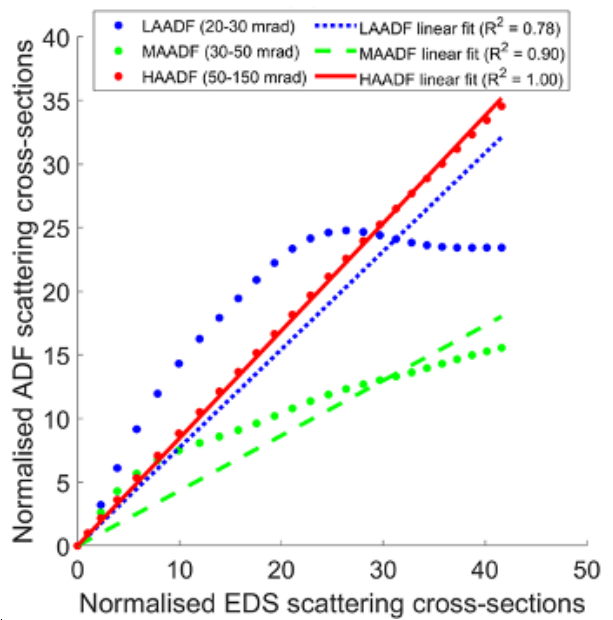

Figure 1. Scattering cross-sections of ADF (with different collection angles) against EDX with linear regression, where the coefficient of determination R2 of EDX-ADF is a measure of linear dependence between different signal modes. The simulations were performed for a Au crystal in [001] direction with varying thicknesses (1-25 atoms), illuminated using $300 \mathrm{keV}$ electrons with a $20 \mathrm{mrad}$ condenser aperture and no lens aberrations.

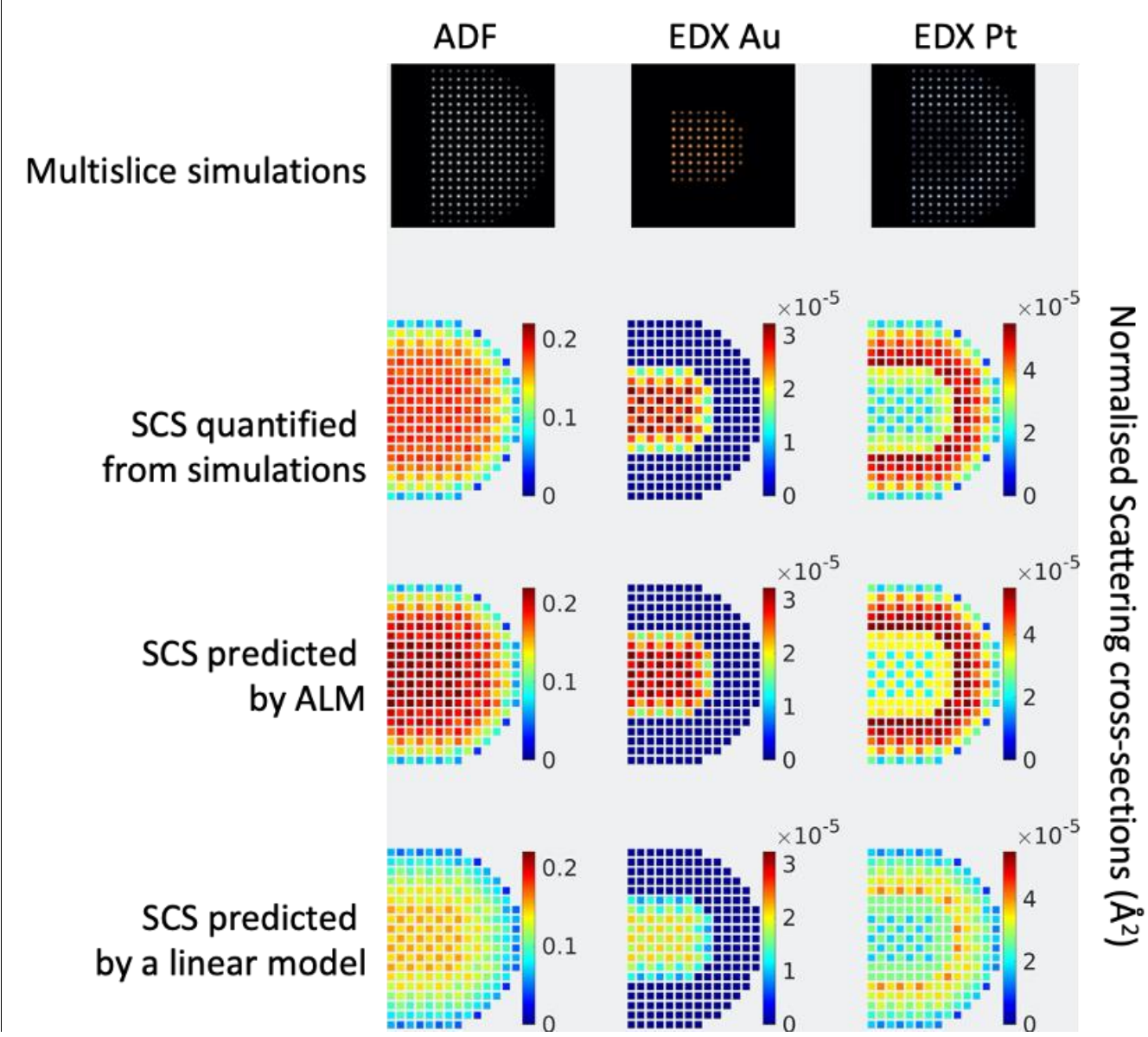

Figure 2. Comparing the simulated and predicted scattering cross-sections (SCS) of ADF and EDX for an $\mathrm{Au}-\mathrm{Pt}$ core-shell nanorod. 


\section{References}

[1] J.M. LeBeau, S.D. Findlay, L.J. Allen, S. Stemmer, Nano Letters 10 (2010) p. 4405-4408.

[2] A. De Backer, K.H.W. van den Bos, W. Van den Broek, J. Sijbers, and S. Van Aert, Ultramicroscopy 171 (2016) p. 104-116.

[3] K.H.W. van den Bos, A. De Backer, G.T. Martinez, N. Winckelmans, S. Bals, P.D. Nellist, S. Van Aert, Physical Review Letters 116 (2016) p. 246101.

[4] A. Varambhia, L. Jones, A. London, D. Ozkaya, P.D. Nellist, \& S. Lozano-Perez, Micron 113 (2018) p. 69-82.

[5] The authors acknowledge financial support from the Research Foundation Flanders (FWO, Belgium) through Project No. G.0502.18N and a post-doctoral grant to ADB. This project has received funding from the European Research Council (ERC) under the European Union's Horizon 2020 research and innovation programme (Grant Agreement No. 770887 PICOMETRICS and No. 823717 ESTEEM3). 